\title{
Individualised versus standard duration of elastic compression therapy for prevention of post- thrombotic syndrome (IDEAL DVT): a multicentre, randomised, single-blind, allocation-concealed, non- inferiority trial
}

Citation for published version (APA):

ten Cate-Hoek, A. J., Amin, E. E., Bouman, A. C., Meijer, K., Tick, L. W., Middeldorp, S., Mostard, G. J. M., ten Wolde, M., van den Heiligenberg, S. M., van Wissen, S., van de Poel, M. H. W., Villalta, S., Serne, E. H., Otten, H-M., Klappe, E. H., Bistervels, I. M., Lauw, M. N., Piersma-Wichers, M., Prandoni, P., ... IDEAL DVT Investigators (2018). Individualised versus standard duration of elastic compression therapy for prevention of post-thrombotic syndrome (IDEAL DVT): a multicentre, randomised, single-blind, allocation-concealed, non-inferiority trial. The Lancet Haematology, 5(1), 25-33. https://doi.org/10.1016/S2352-3026(17)30227-2

Document status and date:

Published: 01/01/2018

DOI:

10.1016/S2352-3026(17)30227-2

Document Version:

Publisher's PDF, also known as Version of record

\section{Document license:}

Taverne

Please check the document version of this publication:

- A submitted manuscript is the version of the article upon submission and before peer-review. There can be important differences between the submitted version and the official published version of record. People interested in the research are advised to contact the author for the final version of the publication, or visit the DOI to the publisher's website.

- The final author version and the galley proof are versions of the publication after peer review.

- The final published version features the final layout of the paper including the volume, issue and page numbers.

Link to publication

\footnotetext{
General rights rights.

- You may freely distribute the URL identifying the publication in the public portal. please follow below link for the End User Agreement:

www.umlib.nl/taverne-license

Take down policy

If you believe that this document breaches copyright please contact us at:

repository@maastrichtuniversity.nl

providing details and we will investigate your claim.
}

Copyright and moral rights for the publications made accessible in the public portal are retained by the authors and/or other copyright owners and it is a condition of accessing publications that users recognise and abide by the legal requirements associated with these

- Users may download and print one copy of any publication from the public portal for the purpose of private study or research.

- You may not further distribute the material or use it for any profit-making activity or commercial gain

If the publication is distributed under the terms of Article $25 \mathrm{fa}$ of the Dutch Copyright Act, indicated by the "Taverne" license above,

Download date: 26 Apr. 2023 


\title{
Individualised versus standard duration of elastic compression therapy for prevention of post-thrombotic syndrome (IDEAL DVT): a multicentre, randomised, single-blind, allocation-concealed, non-inferiority trial
}

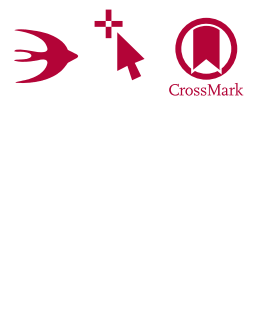

\begin{abstract}
Arina J ten Cate-Hoek, Elham E Amin, Annemieke C Bouman, Karina Meijer, Lidwine W Tick, Saskia Middeldorp, GuyJ M Mostard,
Marije ten Wolde, Simone M van den Heiligenberg, Sanne van Wissen, Marlène HW van de Poel, Sabina Villalta, Erik H Serné, Hans-Martin Otten, Edith H Klappe, Ingrid M Bistervels, Mandy N Lauw, Margriet Piersma-Wichers, Paolo Prandoni, Manuela A Joore, Martin H Prins, Hugo ten Cate, for the IDEAL DVT investigators*
\end{abstract}

\section{Summary}

Background Therapy with elastic compression stockings has been the cornerstone for prevention of post-thrombotic syndrome for decades in patients after acute deep venous thrombosis. It is uncertain who benefits most from therapy, and what the optimum duration of therapy should be. We therefore aimed to assess the safety and efficacy of individualised duration of compression therapy versus the standard duration of 24 months following an initial treatment period of 6 months.

Methods We did a multicentre, randomised, single-blind, allocation-concealed, non-inferiority trial at 12 hospitals in the Netherlands and two in Italy. We randomly assigned patients (1:1) with acute proximal deep vein thrombosis of the leg and without pre-existent venous insufficiency (Clinical Etiological Anatomical and Pathophysiological score $<\mathrm{C} 3$ ) to receive either individualised duration of elastic compression therapy or standard duration of therapy for 24 months following an initial treatment period of 6 months. Randomisation was done with a web-based automatic randomisation programme (TENALEA) and a random block size (2-12), and was stratified by centre, age, and body-mass index. In the initial phase, compression was applied within $24 \mathrm{~h}$ of diagnosis according to three prespecified protocols. All patients received elastic compression stockings $(30-40 \mathrm{~mm} \mathrm{Hg}$ ) for 6 months, and were instructed to wear them every day during ambulant hours. Thereafter treatment was tailored on the basis of clinical signs and symptoms scored according to the Villalta post-thrombotic syndrome scale; patients assigned to individualised therapy with two consecutive Villalta scores of 4 or less were instructed to stop using the stockings. Patients were followed up for 2 years and assessed at five clinic visits at study inclusion, and 3, 6, 12, and 24 months after diagnosis (stocking allocation was not revealed to the assessors). The primary outcome was the proportion of patients with post-thrombotic syndrome at 24 months diagnosed according to original Villalta criteria (a score of $\geq 5$ on two consecutive occasions at least 3 months apart) assessed by intention to treat. The predefined non-inferiority margin for the difference in success rates was set at 7.5\%. This study has been completed and is registered with ClinicalTrials.gov, number NCT01429714.

Findings Between March 22, 2011, and July 1, 2015, we enrolled 865 patients and randomly assigned 437 to individualised duration compression stockings and 428 to standard duration. $283(66 \%)$ of 432 patients in the intervention group were advised before 24 months to stop wearing elastic compression stockings (236 [55\%] of 432 patients after 6 months, and 47 [11\%] of 432 at 12 months). Post-thrombotic syndrome occurred in 125 (29\%) of 432 patients receiving individualised duration of therapy and in $118(28 \%)$ of 424 receiving standard duration of therapy (odds ratio for difference 1.06 , 95\% CI 0.78 to 1.44). The absolute difference was $1 \cdot 1 \%$ (95\% CI $-5 \cdot 2$ to $7 \cdot 3$ ), thus meeting the non-inferiority margin. 24 patients died, $17(4 \%)$ in the individualised treatment group and seven $(2 \%)$ in the standard duration group, but no deaths were related to treatment. No serious adverse events related to the intervention occurred.

Interpretation Individualised therapy with elastic compression stockings for the prevention of post-thrombotic syndrome was non-inferior to standard duration of therapy of 24 months. Individualising the duration is effective and could shorten the length of therapy needed, potentially enhancing patients' wellbeing.

Funding ZonMw (Netherlands).

\section{Introduction}

Post-thrombotic syndrome is a common and chronic complication of deep vein thrombosis of the leg affecting up to half of all patients. ${ }^{1}$ It is characterised by a combination of problems in the leg including tiredness of the leg, pain and oedema, and irreversible skin changes such as hyperpigmentation, venous ectasia, and in more severe cases lipodermatosclerosis and venous ulceration..$^{1-3}$ Post-thrombotic syndrome negatively affects quality of life and is associated with substantial costs. ${ }^{46}$ Once the
Lancet Haematol 2018 5: e25-33

Published Online December 4, 2017 http://dx.doi.org/10.1016/ S2352-3026(17)30227-2

See Comment page e4

*Investigators listed in the appendix

Department of Vascular Medicine, Heart and Vascular Center, Maastricht University Medical Center, Maastricht Netherlands (A) ten Cate-Hoek MD, EE Amin MD, A C Bouman MD, Prof $\mathrm{H}$ ten (ate MD); Department of Hematology, Groningen University Medical Center, Groningen,

Netherlands (Prof K Meijer MD M Piersma-Wichers MD); Department of Interna Medicine, Maxima Medical Center, Veldhoven, Netherlands (LWTick MD); Department of Vascular Medicine, Academic Medical Center, University of Amsterdam, Amsterdam, Netherlands (Prof S Middeldorp MD, $M$ ten Wolde MD, $S$ van Wissen MD I M Bistervels MD, M N Lauw MD); Department of Vascular Medicine, Zuyderland Medical Center, Sittard, Netherlands

(G J M Mostard MD); Department of Medicine, Flevo Hospital, Almere, Netherlands (M ten Wolde, I M Bistervels); Department of Medicine, Westfriesgasthuis, Hoorn, Netherlands

(S M van den Heiligenberg MD), Department of Interna Medicine, Onze Lieve Vrouwe Gasthuis, Amsterdam, Netherlands (S van Wissen); Department of Hematology, 
Laurentius Hospital, Roermond, Netherlands (M H W van de Poel MD); Department of Internal Medicine, Cà Foncello University Hospital, Treviso, Italy (S Villalta MD); Department of Internal Medicine, VU University Medical Center, Amsterdam, Netherlands (E H Serné MD); Department of Internal Medicine, Slotervaart Hospital, Amsterdam, Netherlands (H-M Otten MD); Department of Internal Medicine, Radboud University Medical Center, Nijmegen, Netherlands (E H Klappe MD); Department of Cardiovascular Sciences,

Vascular Medicine Unit,

University of Padua, Padua,

Italy (Prof P Prandoni MD); Department of Clinical Epidemiology and Medical

Technology Assessment, Maastricht University Medical Center, Maastricht, Netherlands (Prof M A Joore PhD, Prof $\mathrm{M} H$ Prins MD); and Laboratory of Clinical Thrombosis and Hemostasis,

Cardio Vascular Research Institute, Maastricht University, Maastricht, Netherlands (Prof $\mathrm{H}$ ten Cate)

Correspondence to:

Dr Arina J ten Cate-Hoek,

Department of Vascular Medicine, Heart and Vascular

Center and Cardiovascular Research Institute, Maastricht

University Medical Center, 5800 NL-6202 AZ Maastricht,

Netherlands

arina.tencate@

maastrichtuniversity.nl

See Online for appendix

\section{Research in context}

\section{Evidence before this study}

We did a systematic review of the scientific literature in September, 2009. We searched MEDLINE, the Current Control Trials database, the UK National Health Service Centre for Reviews and Dissemination database, and the Health Economic Evaluation Database for published articles containing the terms "post thrombotic syndrome" OR "postphlebitic syndrome" AND "compression therapy". We identified five clinical trials, three that showed a strong preventive effect with use of elastic compression stockings initiated in the acute or subacute phase of deep vein thrombosis (DVT) and continued for 2 years. All trials had high adherence rates of more than $80 \%$. Only one trial assessed shortened duration of therapy after initial use of elastic compression stockings and showed no significant benefit for prolonged treatment of 24 months versus treatment for 6 months. Another trial showed no benefit of late initiation of elastic compression stockings 1 year after DVT in patients without complaints and without venous reflux. During our study, the SOX trial (with 803 patients) was published showing no effect of elastic compression stockings compared with sham stockings. Towards the end of our trial the OCTAVIA trial (with 518 patients) was published, highlighting the importance of adherence because it showed that prolonged treatment with elastic compression stockings for another year was not non-inferior in patients without complaints and excellent adherence after 1 year. A meta-analysis reviewing elastic compression stockings initiated in the acute and subacute phase concluded that although there is significant heterogeneity between studies, the overall effect of elastic compression stockings for the prevention of post-thrombotic syndrome is more likely to be in favour of compression, probably more so when only trials with good adherence were assessed. At the time of our study design and initiation, the efficacy of elastic compression stockings for the prevention of post-thrombotic syndrome had been clearly shown and was guideline recommended. However, the optimum duration of treatment was uncertain. Additionally, it was unknown which patients with DVT were likely to benefit most from elastic compression stockings beyond the acute phase. Our study assessed whether individualised duration of treatment with elastic compression stockings beyond the first 6 months was not inferior to standard duration of treatment.

\section{Added value of this study}

We found that it is possible to select patients based on their Villalta score to stop treatment as early as 6 months without increasing the incidence of post-thrombotic syndrome at 24 months. This strategy proved to be highly efficient as treatment could be stopped in $55 \%$ of patients at 6 months and in an additional $11 \%$ of patients at 12 months. No serious adverse events related to individualised duration of treatment occurred.

Implications of all the available evidence

Apart from one negative trial, overall current evidence suggests a beneficial effect of therapy with elastic compression stockings to prevent post-thrombotic syndrome in patients after DVT. Therefore we conclude that individualised therapy duration with elastic compression stockings shortens duration of therapy, is effective, and is likely to reduce health-care costs, although we did not do a formal cost-effectiveness analysis. condition is established, treatment options are scarce. Therefore, preventive therapy with elastic compression stockings has been the cornerstone of treatment for decades since findings from two randomised trials ${ }^{7,8}$ showed that compression therapy for 24 months reduced the risk of post-thrombotic syndrome by approximately $50 \%$.The effectiveness of elastic compression therapy was challenged by findings from the placebo-controlled SOX trial, ${ }^{9}$ which showed no difference between active and placebo stockings. This result affected the clinical community greatly as it gave rise to changes in some guidelines and advice against the routine application of elastic compression therapy. ${ }^{10,11}$ Other groups have been more conservative and did not value the evidence as strong enough to update their recommendations..$^{12,13}$

The SOX trial had several limitations including insufficient adherence to compression therapy, which might have obscured any potential benefit of therapy in subsets of patients..$^{14,15}$ Results from the OCTAVIA trial ${ }^{16}$ showed that in patients with excellent adherence to elastic compression stockings there was less postthrombotic syndrome when the use of stockings was continued beyond 12 months. Hence, OCTAVIA re- emphasised the value of compression therapy, but also reinforced the original question that formed the basis of our study: can the duration of compression therapy be further optimised; ie, tailored on individual basis ${ }^{17,18}$ Tailoring treatment duration to individual needs could retain the effectiveness of compression therapy, enhance patients' wellbeing, and save substantial costs. ${ }^{19}$ We therefore did a study (IDEAL DVT) to compare the effectiveness of individualised duration of elastic compression therapy with standard duration of therapy of 24 months for the prevention of post-thrombotic syndrome. The objective of our study was to assess whether tailoring the duration of elastic compression therapy according to signs and symptoms of an individual patient would not be inferior compared with standard duration of therapy.

\section{Methods}

\section{Study design and participants}

We did a multicentre, randomised, single-blind, allocation-concealed, non-inferiority trial at 14 centres, 12 hospitals in the Netherlands and two in Italy (appendix p 2). This trial was reviewed and approved by 
the institutional review board at each participating centre. The study protocol was published previously. ${ }^{19}$

Eligible participants were adult patients who had an acute, objectively documented proximal thrombosis of the leg and were adequately treated within $24 \mathrm{~h}$ of diagnosis with anticoagulant treatment and initial compression therapy according to one prespecified local study protocol per hospital (appendix p 4). ${ }^{19}$ Patients were ineligible if they had a life expectancy of less than 6 months, underwent active thrombolysis, or had previous ipsilateral deep vein thrombosis of the leg; if pre-existent severe venous insufficiency was present, because signs and symptoms are similar to post-thrombotic syndrome (venous insufficiency was characterised by skin signs C3 to C6 on the Clinical Etiological Anatomical and Pathophysiological [CEAP] score $^{20}$ or by pre-existent requirement of compression therapy); or if they had a contraindication for compression therapy such as intermittent claudication or clinical signs of leg ischaemia. In case of recurrent ipsilateral thrombosis within the first 6 months after diagnosis, patients were excluded from further assessment, as this new event would interfere with the Villalta assessments preceding the intervention and render patients ineligible according to the inclusion criteria. All participants gave written informed consent.

\section{Randomisation and masking}

We randomly assigned eligible patients (1:1) to either individualised duration of elastic compression therapy following an initial treatment period of 6 months followed by individualised extended duration of therapy depending on Villalta scores ${ }^{8,19,21}$ (intervention) or an initial treatment period of 6 months followed by standard duration of therapy for 24 months (control). After obtaining consent, randomisation was done by the coordinating study centre (Maastricht University Medical Center, Maastricht, Netherlands) within 2-6 weeks after the acute onset of deep vein thrombosis through a webbased randomisation programme (TENALEA). A random block size (2-12) was used and randomisation was stratified for centre, age, and body-mass index (BMI). Patients were informed of treatment allocation by telephone by the coordinating study centre. Information on treatment allocation was concealed from the study nurse or the treating physician. Patients were asked to not wear the stockings at the day of their outpatient clinic visits and to not reveal their treatment allocation to the treating physician. Health-care providers were unaware of treatment allocation. Independent clinical research assistants monitored the sites. The investigators collected, maintained, and analysed the data.

\section{Procedures}

A custom-fitted, flat-knitted, knee length graduated compression stocking class III (ankle pressure $30-40 \mathrm{~mm} \mathrm{Hg}$ ) was prescribed for all patients. The pressure class was similar to the pressure used in six of eight previous trials assessing the value of compression in patients with deep venous thrombosis. . $^{7,916,17,22}$ The other two small trials ${ }^{23,24}$ used mixed-treatment modalities.

Compression therapy with knee length elastic compression was started immediately after the initial phase (from diagnosis until resorption of the acute oedema). In the initial phase, compression was applied within $24 \mathrm{~h}$ of diagnosis according to local prespecified protocols. ${ }^{19}$ In the individualised duration group, there were three scenarios: ${ }^{19}$ compression therapy was discontinued if the Villalta scores were 4 or less at both the 3-month and 6-month follow-up visits; compression therapy was continued for the total duration of 24 months if the Villalta score was 5 or more at both visits; or compression therapy was continued for a further 6 months if the Villalta score was 5 or more at the 3-month follow-up visit followed by a score of 4 or less at the 6-month visit. Therapy was then discontinued at the 12-month visit if the score was four or less again; otherwise therapy was continued. If patients did not have two consecutive low scores within 1 year of the acute event, these patients were considered to be chronically unstable and therefore ineligible for shortened treatment duration. If a patient developed symptoms and signs of post-thrombotic syndrome after discontinuation of elastic compression therapy, a predefined protocol was followed (appendix). Treatment was reinstated for a week and then stopped again. In case of persistent symptoms, elastic compression was reinstated permanently.

Patients were followed up for 2 years, during which they visited the outpatient clinic five times: at inclusion into the study and at 3, 6, 12, and 24 months following the diagnosis. At each follow-up visit signs of postthrombotic syndrome were recorded and scored by the study nurse or the treating physician using the objective part of the Villalta clinical scale. ${ }^{21}$ Patients filled out the subjective part of the Villalta scale either in a web-based questionnaire, for which the link was sent to them the day before the planned study visit, or in a questionnaire on paper that was sent to them by regular mail. Prescriptions for new stockings were provided by the coordinating study centre. Adherence to elastic compression therapy was assessed via specific questions in the questionnaires preceding the clinical visits, and in addition by random telephone contacts by study staff. Patients were instructed to contact their physician in case of intermittent leg complaints or signs and symptoms suggestive of recurrent venous thrombosis. Prespecified objective testing was required for these patients. ${ }^{19,25}$ Type and duration of anticoagulation treatment was left at the discretion of the treating physician.

\section{Outcomes}

The primary outcome was the proportion of patients with post-thrombotic syndrome at 24 months after the initial event. Post-thrombotic syndrome was diagnosed with the Villalta scale, as recommended by the subcommittee for standardisation of the International Society on Thrombosis 


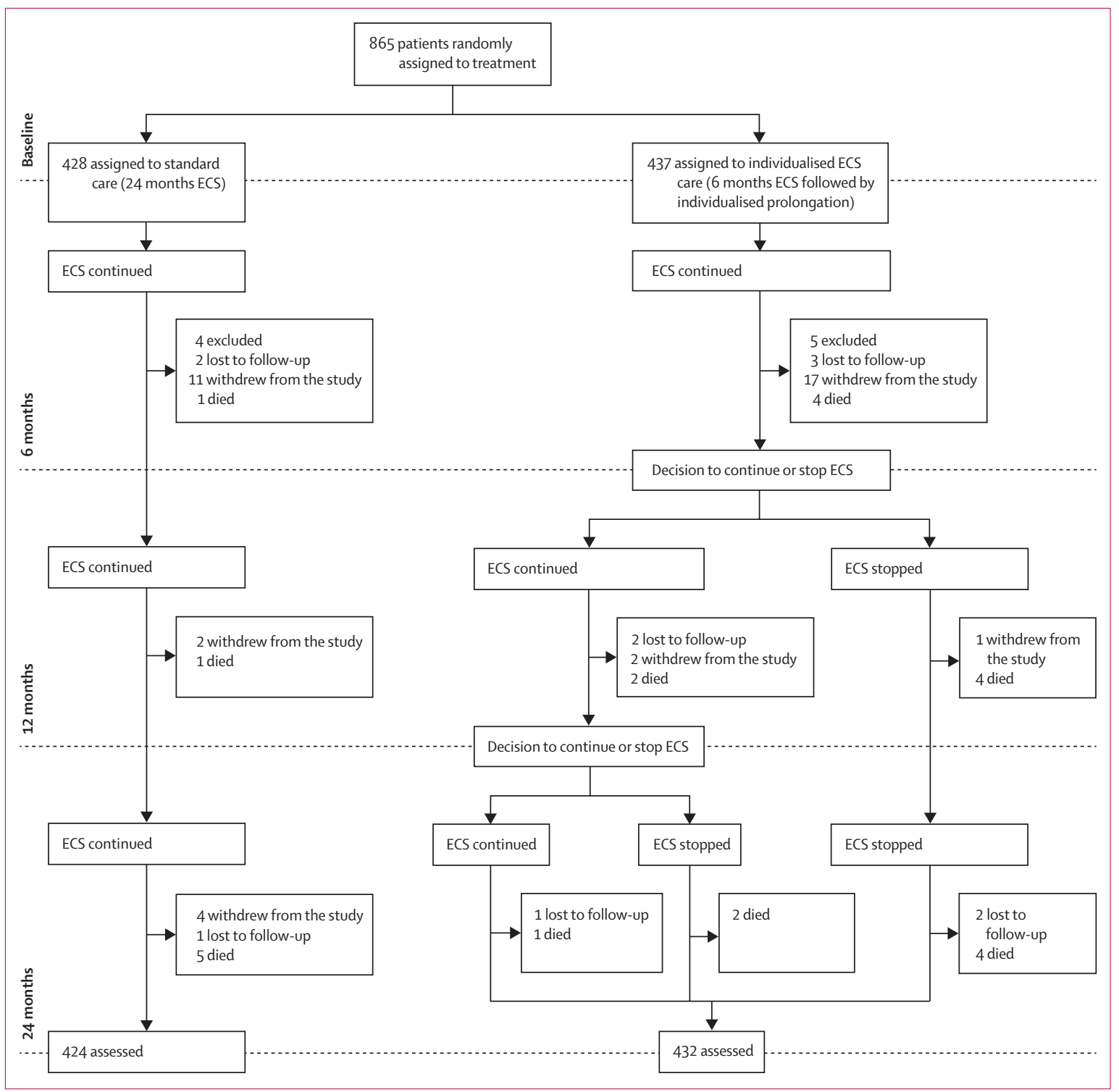

Figure 1: Trial profile

ECS=elastic compression stockings.

and Haemostasis (ISTH). The use of the Villalta scale was promoted as the preferred method of diagnosis for postthrombotic syndrome over other scales for the assessment of venous disease as this scale is disease-specific, widely validated, easy to apply, and sensitive to change. ${ }^{26}$ The scale assesses five patient-rated symptoms: pain, cramps, heaviness, paraesthesia, and itching, and six physical signs: pretibial oedema, skin induration, hyperpigmentation, pain during calf compression, venous ectasia, and redness. All items are graded for intensity on a four-point scale, 0 points when there are no deviations from normal, 1 if the signs or symptoms are mild, 2 if they are moderate, and 3 if they are severe. With 11 items a total score of 33 can be reached, a score of 5-9 is considered mild post-thrombotic syndrome, 10-14 moderate, and a score of 15 or more, or presence of ipsilateral venous ulceration is considered severe post-thrombotic syndrome. ${ }^{2,21}$ We defined postthrombotic syndrome as a Villalta score of 5 or higher at two consecutive visits that were at least 3 months apart (the original scoring method)..$^{8,19,21}$

Additionally, the proportion of patients with postthrombotic syndrome according to the more recently introduced ISTH consensus scoring method (a score of 5 or higher at the 6-month visit or later) was assessed. ${ }^{26}$ The use of the ISTH scoring method results in more patients diagnosed, especially those with mild post-thrombotic syndrome. The original scoring method is more conservative in diagnosing post-thrombotic syndrome. Secondary outcomes included recurrent objectively confirmed venous thromboembolism and venous 
thromboembolism-related death during follow-up assessed by an independent adjudication committee; health-related quality of life measured at each study visit with validated generic questionnaires (SF-36, ${ }^{27}$ EQ-5D ${ }^{28}$ ) and venous disease-specific questionnaires (Dutch translated VEINES-QOL). ${ }^{29,30}$ Two additional secondary outcomes that were indicated in the study protocol are not presented in the current manuscript: patients' preference was assessed at an early stage of the study and was previously published in a separate manuscript, ${ }^{31}$ and costs will be analysed in the context of a cost-effectiveness study.

\section{Statistical analysis}

At a one-sided significance level of 0.05 and a power of $80 \%$, a sample size of 848 was needed in this study to provide sufficient numbers for an adequately powered trial. Loss to follow-up of patients was expected to be less than $2 \%$, thus 864 patients (432 per treatment arm) were needed. The study was designed to show that the effectiveness of individualised duration of elastic compression treatment for the prevention of post-thrombotic syndrome was non-inferior to elastic compression therapy for a standard duration of 24 months. We determined that preserving $70 \%$ of the therapeutic effect would allow a difference of $7.5 \%$ (the non-inferiority margin) in the outcome between the groups. ${ }^{19,32}$ The therapeutic effect of elastic compression therapy for the standard duration of 24 months was estimated from two studies that showed a mean reduction of post-thrombotic syndrome of $26 \%$ using the original Villalta scoring method or a comparable scoring method. ${ }^{7,83}$ For the primary outcome, the proportion of patients with post-thrombotic syndrome at 24 months after the deep vein thrombosis, a univariate analysis of proportions was done with logistic regression $\left(\chi^{2}\right)$ analysis, and odds ratios (OR)s and 95\% CI were calculated. The efficacy analysis included all randomly assigned patients who fitted the inclusion criteria-ie, excluding those who had a recurrent deep vein thrombosis within the first 6 months - and provided written informed consent (intention-to-treat population). Additionally, we used the Kaplan-Meier method to calculate the cumulative incidence of post-thrombotic syndrome, adjusted for centre to compare incidence rates between the two treatment arms. Loss to follow-up, withdrawals, and deaths were censored at the last available date. Hazard ratios (HRs) and 95\% CI were calculated using Cox proportional hazards models, stratified for centre and adjusted for age, sex, and BMI, both for original Villalta scoring and ISTH consensus scoring method. ${ }^{21}$

We did descriptive analyses to assess patient characteristics, and to assess the severity of post-thrombotic syndrome, adherence to therapy, the proportion of recurrent venous thromboembolism (deep vein thrombosis of the leg and pulmonary embolism), and the proportion of (thrombosis-related) death. We applied a mixed-design analysis of variance to test for differences

\begin{tabular}{|c|c|c|}
\hline & $\begin{array}{l}\text { Individualised duration } \\
\text { stockings }(n=437)\end{array}$ & $\begin{array}{l}\text { Standard duration } \\
\text { stockings }(n=428)\end{array}$ \\
\hline Age (years) & $58 \cdot 1(14 \cdot 6)$ & $56 \cdot 3(15 \cdot 7)$ \\
\hline \multicolumn{3}{|l|}{ Age category (years) } \\
\hline$<40$ & $36(8 \%)$ & $53(12 \%)$ \\
\hline $40-65$ years & $255(58 \%)$ & $257(60 \%)$ \\
\hline$>65$ years & $146(33 \%)$ & $117(27 \%)$ \\
\hline \multicolumn{3}{|l|}{ Sex } \\
\hline Men & $253(58 \%)$ & $251(59 \% \%)$ \\
\hline Women & $184(42 \%)$ & $177(41 \%)$ \\
\hline Body-mass index $\left(\mathrm{kg} / \mathrm{m}^{2}\right)$ & $28.0(5 \cdot 1)$ & $27 \cdot 8(5 \cdot 4)$ \\
\hline \multicolumn{3}{|l|}{ Body-mass index category } \\
\hline$<26 \mathrm{~kg} / \mathrm{m}^{2}$ & $172(39 \%)$ & $186(43 \%)$ \\
\hline$\geq 26 \mathrm{~kg} / \mathrm{m}^{2}$ & $261(60 \%)$ & $238(56 \%)$ \\
\hline Previous DVT contralateral & $47(11 \%)$ & $34(8 \%)$ \\
\hline \multicolumn{3}{|l|}{ Characteristics of current DVT } \\
\hline Unprovoked DVT & $295(68 \%)$ & $290(68 \%)$ \\
\hline Right & $191(44 \%)$ & $191(45 \%)$ \\
\hline Left & $231(53 \%)$ & $223(52 \%)$ \\
\hline Bilateral & $2(<1 \%)$ & $2(<1 \%)$ \\
\hline \multicolumn{3}{|l|}{ Most proximal extent of DVT* } \\
\hline Popliteal vein & $215(49 \%)$ & $211(49 \%)$ \\
\hline Femoral vein & $114(26 \%)$ & $122(29 \%)$ \\
\hline Common femoral vein & $96(22 \%)$ & $90(21 \%)$ \\
\hline DVT treatment &.$\cdot$ &.$\cdot$ \\
\hline Vitamin $\mathrm{K}$ antagonist $\dagger$ & $400(92 \%)$ & $378(88 \%)$ \\
\hline Non-vitamin-K anticoagulantsł & $10(2 \%)$ & $18(4 \%)$ \\
\hline Investigational anticoagulants $\$$ & $12(3 \%)$ & $12(3 \%)$ \\
\hline Low-molecular-weight heparin & $15(3 \%)$ & $20(5 \%)$ \\
\hline \multicolumn{3}{|c|}{$\begin{array}{l}\text { Data are mean (SD) or } n(\%) \text {. DVT=deep vein thrombosis, *In the case of bilateral DVT, the leg with the most proximal } \\
\text { DVT was considered to be the index leg. †All patients receiving a vitamin K antagonist had initially } 5-10 \text { days of } \\
\text { low-molecular-weight heparin. } \ddagger \text { The only non-vitamin-K anticoagulant used during the study was rivaroxaban. } \\
\text { \$Some patients participated in studies comparing investigational anticoagulants (warfarin vs edoxaban) and } \\
\text { low-molecular-weight heparin in patients with malignancy. }\end{array}$} \\
\hline
\end{tabular}

between the two treatment groups, and to assess changes over time by comparing repeated outcome measures for quality of life scores at different timepoints of follow-up. The minimally important difference (MID) for quality of life scores were: at least 4 points for the VEINES-QOL T score, ${ }^{34}$ at least 0.07 for the EQ-5D, and at least 0.05 for the SF-36. ${ }^{35}$ For the VEINES-QOL intrinsic score, a MID of at least $0.07\left(0.5\right.$ times the SD) was assumed. ${ }^{36}$ We did a post-hoc analysis to compare the incidence of postthrombotic syndrome at 24 months for patients with a Villalta score of less than 5 at 6 months in the individualised treatment group (stop compression) and the standard treatment group (continue compression). An intention-totreat analytical approach was used for all outcomes. For all analyses a two-sided p value of 0.05 or less was considered significant. Analyses were done with SPSS software, version 23. An independent adjudication committee adjudicated causes of death in the study. This study is registered with ClinicalTrials.gov, number NCT01429714. 


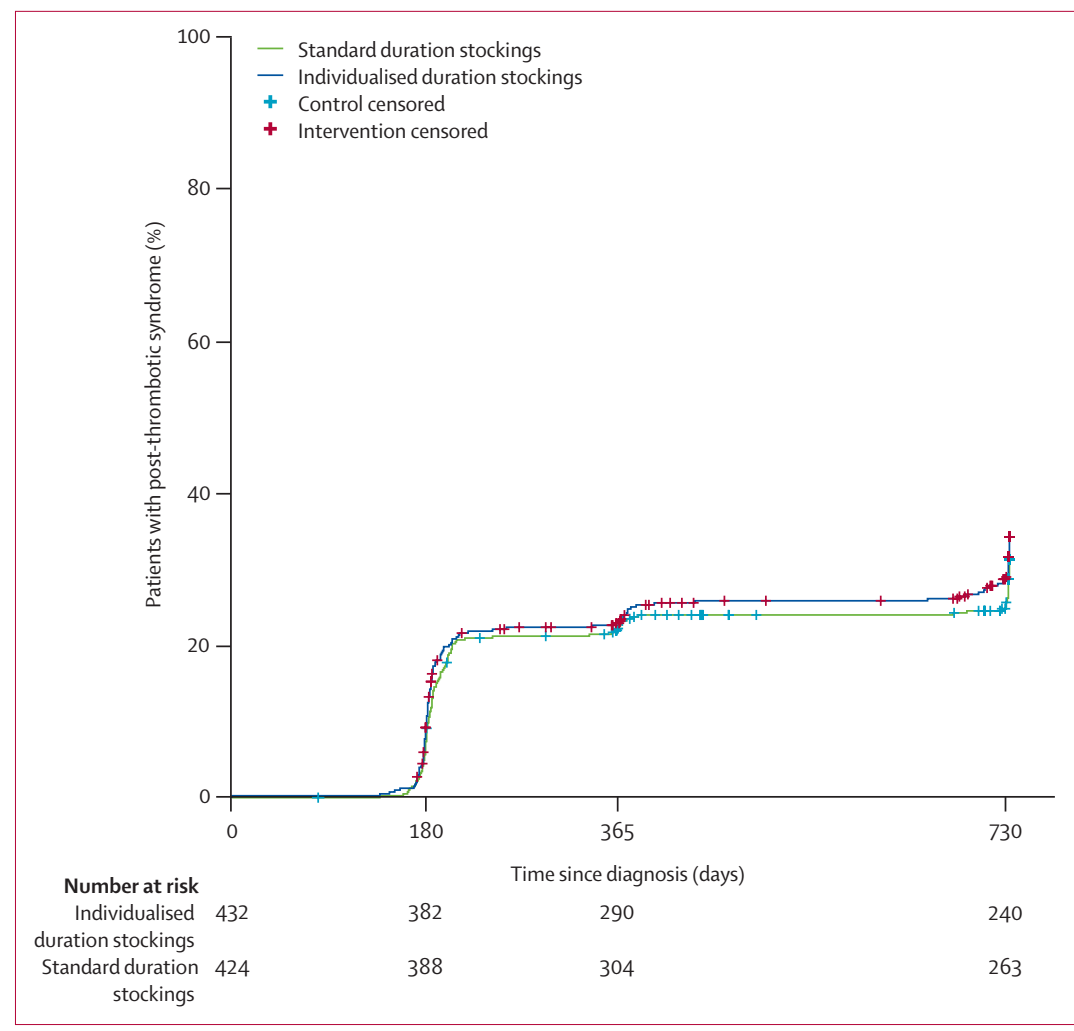

Figure 2: Cumulative incidence of post-thrombotic syndrome

The primary outcome of post-thrombotic syndrome (PTS) according to the original Villalta scoring method was first assessed at the 3-month visit and thereafter at 6, 12, and 24 months. Data from 31 patients who withdrew and four who were lost to follow-up were censored at the time of their last assessment.

\section{Role of the funding source}

The funder of the study has no involvement in study design, study conduct, data collection, data analysis, data interpretation, or writing of the report. The corresponding author had full access to all the data in the study and had final responsibility for the decision to submit for publication.

\section{Results}

Between March 22, 2011, and July 1, 2015, we enrolled 865 patients and randomly assigned 437 to individualised duration of elastic compression therapy and 428 to standard duration of therapy. Patients were included in the study within a median of 18 days (IQR 17-19) of the diagnosis of acute proximal deep vein thrombosis; however, predefined compression treatment started within $24 \mathrm{~h}$ of the diagnosis (appendix p 4). After the exclusion of nine patients because of a recurrent thrombotic event of the leg within the first 6 months after enrolment, 856 patients were included in the primary intention-totreat analysis (figure 1). Baseline characteristics and anticoagulant treatment were similar for both groups (table 1). $283(66 \%)$ of 432 patients in the intervention group were advised before 24 months to stop wearing elastic compression stockings (236 [55\%] of 432 patients after 6 months, and $47[11 \%]$ of 432 at 12 months). In nine (3\%) of
283 patients therapy was reinitiated and permanently reinstated because of persistent leg complaints. In another eight (3\%) of 283 patients elastic compression therapy was temporarily reinstated for a week, according to protocol. Adherence to treatment advice was similar in both groups with either daily or almost daily use, or no use of stockings as appropriate in 383 (96\%) of 400 patients at 3 months, $369(93 \%)$ of 395 at 6 months, $286(81 \%)$ of 354 at 12 months and 240 (77\%) of 313 at 24 months in patients receiving individualised treatment and with daily or almost daily use of stockings in 382 (96\%) of 399, 363 (93\%) of 390, $328(87 \%)$ of 377, and $269(79 \%)$ of 340 patients receiving standard therapy (appendix $p$ 11). The mean percentage of adherence registered with random telephone calls throughout the follow-up was 380 (SD 8.0; $88 \%)$ of 432 for the intervention group and $302(49 \cdot 7$; $71 \%$ ) of 424 in the control group.

Post-thrombotic syndrome occurred in 125 (29\%) of 432 patients receiving individualised duration of therapy and $118(28 \%)$ of 424 patients who received standard duration of therapy (OR 1.06, 95\% CI, 0.78-1.44) (figure 2, table 2). The absolute difference was $1.1 \%$ (95\% CI $-5 \cdot 2$ to $7 \cdot 3)$. The cumulative incidence of post-thrombotic syndrome at 730 days was $28 \cdot 9 \%(95 \%$ CI $24 \cdot 7-33 \cdot 5)$ in the individualised duration group versus $27 \cdot 8 \%(23 \cdot 6-32 \cdot 4)$ in the standard duration group (hazard ratio [HR] adjusted for centre $1 \cdot 13,95 \%$ CI $0 \cdot 88-1 \cdot 46 ; p=0 \cdot 77)$. Distribution of severity of post-thrombotic syndrome was similar for both groups. The analysis according to the ISTH-scoring method showed higher incidences but similar relative efficacy (table 2). Per-protocol analysis showed similar results (appendix p 12).

The cumulative incidence of post-thrombotic syndrome for individualised treatment duration and standard treatment duration showed that most cases were identified at 6 months (87 [21\%] of 424 and 92 [21\%] of 432, respectively) and that thereafter there was a slight increase at 12 months (ten [2\%] of 424 and eight [2\%] of 432), and 24 months (21 [5\%] of 424 and 25 [6\%] of 432, according to the original scoring method). The proportion of patients with recurrent venous thromboembolic episodes was similar for both groups, and mortality was higher in the individualised treatment duration group (table 2). The HRs and 95\% CI stratified for centre and adjusted for age, sex, and BMI category (cutoff 26) for the original Villalta scoring and the ISTH-consensus scoring method were $1.13(0 \cdot 88-1 \cdot 46)$ and $1 \cdot 15(0 \cdot 94-1 \cdot 40)$, respectively.

24 patients died, but no deaths were related to treatment (table 2). Most deaths in both groups were related to cancer. No serious adverse events related to the intervention occurred.

Post-hoc analysis (including 420 patients) using the ISTH-scoring method showed an incidence of postthrombotic syndrome for individualised treatment in the group of early stoppers (those with Villalta score $<5$ at 6 months) of $36 \%$ (45 of 207 patients) compared with 
$22 \%$ (76 of 213 patients) for the group that continued use of elastic compression stockings for 24 months (standard duration) with an absolute difference in incidence of post-thrombotic syndrome of $14.0 \%$; relative risk (RR) 1.6 (95\% CI 1.2-2.3); number-needed-to-treat $7 \cdot 2(4 \cdot 4-20 \cdot 7)$. When the original scoring method was employed (including 419 patients) the differences were non-significant with an incidence of post-thrombotic syndrome of $10 \%$ (21 of 212 patients) in the early stoppers versus $7 \%$ (14 of 207 patients) for those that continued treatment (RR 1.52, 95\% CI 0.71-2.97).

Generic quality of life was similar in both treatment groups: SF-36 ranged from 0.62 at 1 month to 0.82 at 24 months in patients with individualised treatment versus 0.61 to 0.82 for patients with standard therapy, $\mathrm{p}=0.86$. EQ-5D ranged from 0.82 to 0.90 in patients receiving individualised treatment versus 0.83 to 0.88 in the standard treatment group, $\mathrm{p}=0 \cdot 51$. Disease-specific quality-of-life measures were also similar between groups: VEINES-QOL T scores ranged from $50 \cdot 3$ at 1 month to 50.0 at 24 months for patients with individualised treatment versus 49.8 to 50.0 in patients with standard treatment, $p=0 \cdot 34$. The VEINES-QOL intrinsic score ranged from 0.63 to 0.76 in patients with individualised treatment versus 0.63 to 0.75 in patients with standard therapy, $\mathrm{p}=0.64$ (appendix p 13). Except for the VEINESQOL T score $(\mathrm{p}=0 \cdot 88)$, all QOL measurements significantly increased over time $(\mathrm{p}<0 \cdot 0001)$. These differences were clinically important for SF-36 and for the VEINESQOL intrinsic score in both groups. For the EQ5D score the MID of 0.07 or greater occurred in the individualised treatment group only.

\section{Discussion}

Our study findings showed that individualised duration of elastic compression therapy is non-inferior to standard duration of treatment. Elastic compression stockings are commonly viewed as unattractive and uncomfortable; however, the expected positive effect of shorter duration of elastic compression on quality of life could not be substantiated; quality of life between treatment groups was similar for all measures, and improved over time for most measures. The observed incidence of post-thrombotic syndrome was similar to incidences reported for standard duration of elastic compression in previous landmark trials by Brandjes and colleagues ${ }^{7}$ and Prandoni and colleagues, ${ }^{8}$ indicating that individualised duration of elastic compression therapy is feasible without increasing the incidence of post-thrombotic syndrome. Moreover, individualised duration of treatment was efficient, as more than half the patients in the intervention group could stop wearing the stockings as early as 6 months after the thrombosis and in only $3 \cdot 2 \%$ of cases was permanent reinstatement of treatment necessary.

The need for elastic-compression therapy was previously challenged by findings from the SOX trial ${ }^{9}$ that showed an unexpected absence of efficacy with elastic compression

\begin{tabular}{|c|c|c|c|}
\hline & $\begin{array}{l}\text { Individualised } \\
\text { duration compression } \\
\text { stockings }(n=432)\end{array}$ & $\begin{array}{l}\text { Standard duration } \\
\text { compression } \\
\text { stockings }(n=424)\end{array}$ & $\begin{array}{l}\text { Odds ratio } \\
(95 \% \mathrm{Cl})\end{array}$ \\
\hline \multicolumn{4}{|l|}{ Primary outcome } \\
\hline $\begin{array}{l}\text { Number of post-thrombotic events } \\
\text { assessed by Villalta according to original } \\
\text { scoring* } 24 \text { months after DVT }\end{array}$ & $125(29 \%)$ & $118(28 \%)$ & $1.06(0.78-1.44)$ \\
\hline \multicolumn{4}{|l|}{ Villalta severity category (scores) } \\
\hline None $(<5)$ & $307(71 \%)$ & $305(72 \%)$ &.. \\
\hline Mild (5-9) & $84(19 \%)$ & $67(16 \%)$ &.. \\
\hline Moderate (10-14) & $32(7 \%)$ & $35(8 \%)$ &.. \\
\hline Severe (>14 or ulcer) & $9(2 \%)$ & $17(4 \%)$ &.. \\
\hline \multicolumn{4}{|l|}{ Secondary outcomes } \\
\hline $\begin{array}{l}\text { Number of post-thrombotic events } \\
\text { assessed by Villalta's criteria according to } \\
\text { ISTH scoring }+24 \text { months after DVT }\end{array}$ & $219(51 \%)$ & $192(45 \%)$ & $1.24(0.94-1.64)$ \\
\hline \multicolumn{4}{|l|}{ Villalta severity category (scores) } \\
\hline None $(<5)$ & $213(49 \%)$ & $233(55 \%)$ &.. \\
\hline Mild (5-9) & $177(41 \%)$ & $142(33 \%)$ &.. \\
\hline Moderate (10-14) & $35(8 \%)$ & $35(8 \%)$ &.. \\
\hline Severe (>14 or ulcer) & $7(2 \%)$ & $14(3 \%)$ &. \\
\hline Ipsilateral leg ulcer & $2(<1 \%)$ & 0 &.. \\
\hline Recurrent DVT & $21(5 \%)$ & $27(6 \%)$ &.. \\
\hline Recurrent pulmonary embolism & $13(3 \%)$ & $9(2 \%)$ &.. \\
\hline Death $\neq$ & $17(4 \%)$ & $7(2 \%)$ &.. \\
\hline Malignancy related & $12(3 \%)$ & $5(1 \%)$ &.$\cdot$ \\
\hline Documented venous thromboembolism & 0 & 0 &. \\
\hline $\begin{array}{l}\text { Venous thromboembolism cannot be } \\
\text { excluded }\end{array}$ & $3(<1 \%)$ & $1(<1 \%)$ &.. \\
\hline Other & $2(<1 \%)$ & $1(<1 \%)$ &.. \\
\hline \multicolumn{4}{|c|}{$\begin{array}{l}\text { Of the } 865 \text { patients in the intention-to-treat analysis, } 856 \text { were included in the time-to-event analysis. Nine patients } \\
\text { (four in the standard group and five in the intervention group) were excluded because of recurrent venous thrombosis in } \\
\text { the leg within the first } 6 \text { months. DVT=deep vein thrombosis. ISTH=International Society on Thrombosis and } \\
\text { Hemostasis. *Original scoring:Villalta score of at least } 5 \text { at two consecutive visits at least } 3 \text { months apart. HISTH scoring: } \\
\text { Villalta score of at least } 5 \text { or an ulcer after the } 6 \text {-month visit. } ¥ \text { See appendix for individual causes of death (p 9). }\end{array}$} \\
\hline
\end{tabular}

therapy for the prevention of post thrombotic syndrome. Non-adherence to therapy was identified as a plausible and important contributor to the negative findings of SOX. ${ }^{14}$ Apart from this one large negative trial, overall evidence suggests that elastic compression therapy is effective for around a $30 \%$ reduction of post-thrombotic syndrome. . $^{1,25,37,38}$ Findings from the OCTAVIA trial have shown that in selected patients with excellent adherence $(85 \%$ of patients using elastic compression stockings for 6-7 days a week), outcomes after stopping treatment at 12 months were inferior to standard duration of treatment, with 14 needed to treat for the extra year for the prevention of postthrombotic syndrome-highlighting the importance of therapy adherence for efficacy. ${ }^{16}$ In our study, adherence to therapy advice was high throughout the study (more than $90 \%$ after 3 months and almost $80 \%$ at the last follow-up visit). These adherence values are similar to those reported in other trials that showed efficacy of compression therapy. ${ }^{7,816}$ A post-hoc analysis of data from our IDEAL study comparing cessation of stockings in patients in the 
individualised treatment group with Villalta scores less than 5 with similar patients in the standard treatment group who continued stocking therapy showed a significant benefit in the standard treatment group, with seven needed to treat for the continuation of 18 more months therapy for the prevention of post-thrombotic syndrome when using the ISTH scoring method. However, there were no significant between-group differences when the original scoring method was used. This result indicates that the treatment effect shown in the OCTAVIA trial might be directed at mild post-thrombotic syndrome. The conclusion could be that compression after around 6 or 12 months benefits mainly patients with mild postthrombotic syndrome caused by oedema. Whether it is cost-effective in the long run to provide such patients with elastic compression stockings and thus prevent further damage due to venous hypertension, or whether postthrombotic syndrome in these patients is so mild that therapy may be forgone, should be assessed by a formal cost-effectiveness analysis to estimate the long-term costs and effects of this strategy.

From a pathophysiological point of view, compression in the (sub) acute phase would be expected to be more effective for prevention of post-thrombotic syndrome than compression at a later stage. Compression counteracts the acute increase of venous pressure caused by the obstructing thrombus and might thereby prevent secondary skin changes associated with oedema and inflammation. ${ }^{39,40}$ Additionally, compression restores venous flow and improves calf muscle function, which might as a result promote thrombus resolution and reduce vein wall remodelling, and thereby prevent post-thrombotic syndrome..$^{41-43}$

Our study had some potential limitations. First, our study was designed and initiated at a time of grade $1 \mathrm{~A}$ evidence for the efficacy of elastic compression therapy for prevention of post-thrombotic syndrome. ${ }^{44}$ Therefore assessing the effectiveness of elastic compression therapy itself was not defined as one of the study objectives (there is now some uncertainty about the efficacy of elastic compression). Second, definition of a non-inferiority margin is always subjective and the clinical relevance of meeting or not meeting a criterion by just a few percentage points could be debated, when the overall incidence of the event is around $30 \%$. We can, however, be confident that the individualised intervention was truly non-inferior, since the outcomes were numerically similar in both groups. Third, mortality was unevenly distributed between groups, with an imbalance mainly related to malignancy. However, we attributed this result to chance, as there was no biological plausibility for a true effect.

What do these outcomes mean for individual patients and health-care budgets? Individualised shortening of the duration of therapy based on the original Villalta scoring method is an effective and safe strategy that might be less demanding for patients. Additionally, an individualised therapy strategy has the potential to substantially reduce costs without increasing the incidence of moderate to severe post-thrombotic syndrome. Whether continuation of compression beyond 6 months with the intention to prevent mild post-thrombotic syndrome is cost-effective needs to be assessed. To obtain a more precise estimation of its effect on health-care budgets and to assess the long-term consequences of these strategies, a formal cost-effectiveness analysis should be done.

In conclusion, individualised duration of therapy with elastic compression stockings for the prevention of post-thrombotic syndrome was non-inferior to standard duration therapy of 24 months for patients with acute proximal deep vein thrombosis of the leg. Individualised duration of therapy resulted in shortened treatment duration of only 6 months in more than $50 \%$ of patients, and was safe and effective, and is therefore likely to enhance patients' wellbeing and reduce health-care costs.

\section{Contributors}

AJtC-H contributed to study concept, study design, funding, recruitment of centres, data collection, data analysis, data interpretation, and writing of the manuscript. EEA contributed to the data collection, data analysis, data interpretation, and writing of the manuscript. MAJ contributed to the study design, funding, data interpretation, and writing of the manuscript. MHP contributed to study design, funding, data analysis, data interpretation, and writing of the manuscript. HtC contributed to study concept, study design, funding, data collection, data interpretation, and writing of the manuscript. ACB contributed to data collection, data analysis, data interpretation, and writing of the manuscript. All other authors contributed equally to data collection, and writing and reviewing of the manuscript.

\section{Declaration of interests}

We declare no competing interests.

\section{Acknowledgments}

The trial was funded by a grant from ZonMw (Netherlands; grant number 171102007), the Netherlands Organisation for Health Research and Development (a government organisation promoting research into cost-effectiveness of medical treatments). The underlying research protocol for the IDEAL study was reviewed by international reviewers and adapted as appropriate based on their comments during the grant application process. We thank Marianne Nelis, Annemieke Lindl, Rosa van Gorp (Maastricht University Medical Centre, Maastricht, Netherlands), Sandra Walstra, Femke Yspeerd, Marja Voskuilen, Karen Thedinga (Groningen University Medical Centre, Groningen, Netherlands), Marleen Bax (Maxima Medical Centre, Eindhoven, Netherlands), Judith Westra, Yvette Henstra, Roel Blanken (Onze Lieve Vrouwe Gasthuis, Amsterdam, Netherlands), Olga Ternede (Slotervaart Hospital, Amsterdam, Netherlands), Catherine Combee, and Alex Patty (Free University Medical Centre, Amsterdam, Netherlands), and all study supporting staff and nurses of the collaborating centres.

\section{References}

1 Prandoni P, Kahn SR. Post-thrombotic syndrome: prevalence, prognostication and need for progress. Br J Haematol 2009; 145: 286-95.

2 Kahn SR, Shrier I, Julian JA, et al. Determinants and time course of the postthrombotic syndrome after acute deep venous thrombosis. Ann Intern Med 2008; 149: 698-707.

3 Pesavento R, Villalta S, Prandoni P. The postthrombotic syndrome. Intern Emerg Med 2010; 5: 185-92.

4 Lubberts B, Paulino Pereira NR, Kabrhel C, Kuter DJ, DiGiovanni CW. What is the effect of venous thromboembolism and related complications on patient reported health-related quality of life? A meta-analysis. Thromb Haemost 2016; 116: 417-31.

5 Bergqvist D, Jendteg S, Johansen L, Persson U, Odegaard K. Cost of long-term complications of deep venous thrombosis of the lower extremities: an analysis of a defined patient population in Sweden. Ann Intern Med 1997; 126: 454-57. 
6 Kahn SR, Hirsch A, Shrier I. Effect of postthrombotic syndrome on health-related quality of life after deep venous thrombosis. Arch Intern Med 2002; 162: 1144-48.

7 Brandjes DP, Buller HR, Heijboer H, et al. Randomised trial of effect of compression stockings in patients with symptomatic proximal-vein thrombosis. Lancet 1997; 349: 759-62.

8 Prandoni P, Lensing AW, Prins MH, et al. Below-knee elastic compression stockings to prevent the post-thrombotic syndrome: a randomized, controlled trial. Ann Intern Med 2004; 141: 249-56.

9 Kahn SR, Shapiro S, Wells PS, et al. Compression stockings to prevent post-thrombotic syndrome: a randomised placebo-controlled trial. Lancet 2014; 383: 880-88.

10 Kearon C, Akl EA, Ornelas J, et al. Antithrombotic therapy for VTE disease: CHEST Guideline and Expert Panel Report. Chest 2016; 149: $315-52$

11 NICE. Venous Thromboembolism in adults: diagnosis and management, 2015. www.nice.org.uk/guidance/cg144 (accessed Nov 27, 2017).

12 Cardiovascular Disease Educational and Research Trust, European Venous Forum, North American Thrombosis Forum, International Union of Angiology, Union Internationale du Phlebologie. Prevention and treatment of venous thromboembolism: international consensus statement (guidelines according to scientific evidence). Clin Appl Thromb Hemost 2013; 19: 116-18.

13 CBO Richtlijn Diagnostiek. Preventie en behandeling van veneuze trombo-embolie en secundaire preventie van arteriele trombose. Kwaliteitsinstituut voor de Gezondheidszorg CBO. 2008. https://www.nvr.nl/cbo-richtlijnen (accessed Nov 27, 2017).

14 ten Cate-Hoek AJ. Elastic compression stockings-is there any benefit? Lancet 2014; 383: 851-53.

15 Labropoulos N, Gasparis AP, Caprini JA, Partsch H. Compression stockings to prevent post-thrombotic syndrome. Lancet 2014; 384: 129-30.

16 Mol GC, van de Ree MA, Klok FA, et al. One versus two years of elastic compression stockings for prevention of post-thrombotic syndrome (OCTAVIA study): randomised controlled trial. BMJ 2016; 353: i2691.

17 ten Cate-Hoek AJ, ten Cate H, Tordoir J, Hamulyak K, Prins MH. Individually tailored duration of elastic compression therapy in relation to incidence of the postthrombotic syndrome. J Vasc Surg 2010; 52: 132-38.

18 Aschwanden M, Jeanneret C, Koller MT, Thalhammer C, Bucher HC Jaeger KA. Effect of prolonged treatment with compression stockings to prevent post-thrombotic sequelae: a randomized controlled trial. J Vasc Surg 2008; 47: 1015-21.

19 ten Cate-Hoek AJ, Bouman AC, Joore MA, Prins M, ten Cate H. The IDEAL DVT study, individualised duration elastic compression therapy against long-term duration of therapy for the prevention of post-thrombotic syndrome: protocol of a randomised controlled trial. BMJ Open 2014; 4: e005265.

20 Eklof B, Rutherford RB, Bergan JJ, et al. Revision of the CEAP classification for chronic venous disorders: consensus statement. J Vasc Surg 2004; 40: 1248-52.

21 Villalta S, Bagatella P, Piccioli A, Lensing A, Prins M, Prandoni P. Assessment of validity and reproducibility of a clinical scale for the post-thrombotic syndrome Haemostasis 1994; 24: 158a.

22 Aschwanden M, Jeanneret C, Koller MT, Thalhammer C, Bucher HC, Jaeger KA. Effect of prolonged treatment with compression stockings to prevent post-thrombotic sequelae: a randomized controlled trial. J Vasc Surg 2008; 47: 1015-21.

23 Partsch H, Blättler W. Compression and walking versus bed rest in the treatment of proximal deep venous thrombosis with low molecular weight heparin. J Vasc Surg 2000; 32: 861-69.

24 Ginsberg JS, Hirsh J, Julian J, et al. Prevention and treatment of postphlebitic syndrome: results of a 3-part study. Arch Intern Med 2001; 161: 2105-09.

25 Prandoni P, Lensing AW, Bernardi E, Villalta S, Bagatella P, Girolami A. The diagnostic value of compression ultrasonography in patients with suspected recurrent deep vein thrombosis. Thromb Haemost 2002; 88: 402-06.
26 Kahn SR, Partsch H, Vedantham S, Prandoni P, Kearon C. Definition of post-thrombotic syndrome of the leg for use in clinical investigations: a recommendation for standardization. J Thromb Haemost 2009; 7: 879-83.

27 Aaronson NK, Muller M, Cohen PD, et al. Translation, validation, and norming of the Dutch language version of the SF-36 Health Survey in community and chronic disease populations. J Clin Epidemiol 1998; 51: 1055-68.

28 Brooks R. EuroQol: the current state of play. Health Policy 1996 37: $53-72$.

29 Kahn SR, Lamping DL, Ducruet T, et al. VEINES-QOL/Sym questionnaire was a reliable and valid disease-specific quality of life measure for deep venous thrombosis. J Clin Epidemiol 2006; 59: $1049-56$

30 van der Velden SK, Biemans AA, Nijsten T, Sommer A. Translation and validation of the Dutch VEINES-QOL/Sym in varicose vein patients. Phlebology 2014; 29: 227-35.

31 Bouman AC, Ten Cate-Hoek AJ, Dirksen CD, Joore MA. Eliciting patients' preferences for elastic compression stocking therapy after deep vein thrombosis: potential for improving compliance. J Thromb Haemost 2016; 14: 510-17.

32 Rothmann MD, Tsou HH. On non-inferiority analysis based on delta-method confidence intervals. J Biopharm Stat 2003; 13: 565-83.

33 Bouman AC, ten Cate-Hoek AJ, Ramaekers BL, Joore MA. Sample size estimation for non-inferiority trials: frequentis approach versus decision theory approach. PLoS One 2015; 10: e0130531.

34 Lamping DL, Schroter S, Kurz X, Kahn SR, Abenhaim L. Evaluation of outcomes in chronic venous disorders of the leg: development of a scientifically rigorous, patient-reported measure of symptoms and quality of life. J Vasc Surg 2003; 37: 410-19.

35 Walters SJ, Brazier JE. Comparison of the minimally important difference for two health state utility measures: EQ-5D and SF-6D. Qual Life Res 2005; 14: 1523-32.

36 Norman GR, Sloan JA, Wyrwich KW. The truly remarkable universality of half a standard deviation: confirmation through another look. Expert Rev Pharmacoecon Outcomes Res 2004; 4: $581-85$.

37 GitHub contributors. Compression stockings to prevent postphlebitic syndrome, 2017. https://github.com/openMetaAnalysis/ Compression-stockings-to-prevent-post-phlebitic-syndrome/blob/ master/README.md (accessed Nov 27, 2017).

38 Appelen D, van Loo E, Prins MH, Neumann MH, Kolbach DN. Compression therapy for prevention of post-thrombotic syndrome. Cochrane Database Syst Rev 2017; 9: Cd004174.

39 Meissner MH, Wakefield TW, Ascher E, et al. Acute venous disease: venous thrombosis and venous trauma. J Vasc Surg 2007; 46 (suppl S): 25s-53s

40 Perrin M, Ramelet AA. Pharmacological treatment of primary chronic venous disease: rationale, results and unanswered questions. Eur J Vasc Endovasc Surg 2011; 41: 117-25.

41 Cooley BC, Chen CY, Hess R, Schmeling G. Incomplete resolution of deep vein thrombosis under reduced flow conditions. Thromb Res 2013; 131: 55-58

42 Mosti G, Iabichella ML, Partsch H. Compression therapy in mixed ulcers increases venous output and arterial perfusion. J Vasc Surg 2012; 55: 122-28

43 Flour M, Clark M, Partsch $\mathrm{H}$, et al. Dogmas and controversies in compression therapy: report of an International Compression Club (ICC) meeting, Brussels, May 2011. Int Wound J 2013; 10: 516-26.

44 Kearon C, Kahn SR, Agnelli G, Goldhaber S, Raskob GE, Comerota AJ. Antithrombotic therapy for venous thromboembolic disease: American College of Chest Physicians evidence-based clinical practice guidelines (8th edn). Chest 2008; 133 (suppl 6): 454S-545S. 